\title{
Implementation of TAGE Method Using Seikkala Derivatives Applied to Two-Point Fuzzy Boundary Value Problems
}

\author{
A. A. Dahalan ${ }^{1}$ and J. Sulaiman ${ }^{2}$ \\ ${ }^{1}$ Department of Mathematics, Centre for Defence Foundation Studies, National Defence University of Malaysia, \\ 57000 Kuala Lumpur, Malaysia \\ ${ }^{2}$ Faculty of Science \& Natural Resources, Universiti Malaysia Sabah, 88400 Kota Kinabalu, Sabah, Malaysia
}

Correspondence should be addressed to A. A. Dahalan; a.qilah@upnm.edu.my

Received 21 January 2015; Revised 15 April 2015; Accepted 19 April 2015

Academic Editor: Bashir Ahmad

Copyright (C) 2015 A. A. Dahalan and J. Sulaiman. This is an open access article distributed under the Creative Commons Attribution License, which permits unrestricted use, distribution, and reproduction in any medium, provided the original work is properly cited.

\begin{abstract}
Iterative methods particularly the Two-Parameter Alternating Group Explicit (TAGE) methods are used to solve system of linear equations generated from the discretization of two-point fuzzy boundary value problems (FBVPs). The formulation and implementation of the TAGE method are also presented. Then numerical experiments are carried out onto two example problems to verify the effectiveness of the method. The results show that TAGE method is superior compared to GS method in the aspect of number of iterations, execution time, and Hausdorff distance.
\end{abstract}

\section{Introduction}

Fuzzy boundary value problems (FBVPs) and treating fuzzy differential equations were one of the major applications for fuzzy number arithmetic [1]. FBVPs can be approached by two types. For instance, the first approach addresses problems in which the boundary values are fuzzy where the solution is still in fuzzy function. Then the second approach is based on generating the fuzzy solution from the crisp solution [2]. To solve these problems, numerical methods obtain their approximate solution. Consequently, in this paper, let twopoint linear FBVPs be defined in general form as follows:

$$
\begin{aligned}
\tilde{x}^{\prime \prime}(t)+p(t) \tilde{x}^{\prime}(t)+q(t) \tilde{x}(t) & =f(t), \quad t \in[a, b], \\
\widetilde{x}(a) & =\sigma, \\
\widetilde{x}(b) & =\omega,
\end{aligned}
$$

where $\widetilde{x}(t)$ is a fuzzy function and $f(t), p(t)$, and $q(t)$ are continuous functions on $[a, b]$, whereas, $\sigma$ and $\omega$ are fuzzy numbers.

Based on the Seikkala derivative [3], (1) will be solved numerically by applying the second-order central finite difference scheme to discretize the two-point linear FBVPs into linear systems. Then the generated linear systems will be solved iteratively by using Two-Parameter Alternating Group Explicit (TAGE) method $[4,5]$. By considering the Group Explicit (GE) method for the numerical solution of parabolic and elliptic problems, Evans [6,7] discovered Alternating Group Explicit method. Later, Sukon and Evans [5] expanded this approach to initiate the TAGE method thus proving that this method is superior compared to AGE method. From previous studies, findings of the papers related to the TAGE iterative method and its variants [8-13] have shown that TAGE method has been widely used to solve the nonfuzzy problems. Due to the efficiency of the methods, this paper extends the application of TAGE iterative method in solving fuzzy problems. Since the fuzzy linear systems will be constructed, the iterative method becomes the natural option to get a fuzzy numerical solution of the problem.

The outline of the paper is organized as follows. Section 2 will discuss the finite difference method based on the secondorder finite difference scheme in discretizing two-point FBVPs, while Section 3 presents the formulation and implementation of the TAGE methods in solving linear systems generated from the second-order finite difference scheme. Section 4 shows some numerical examples and conclusions are given in Section 5. 


\section{Finite Difference Approximation Equations}

To be clear, let $\tilde{x}$ be a fuzzy subset of real numbers. It is characterized by the corresponding membership function evaluated at $t$, writing $\tilde{x}(t)$ as a number in $[0,1]$. $\alpha$-cut of $\tilde{x}$, in which $\alpha$ is denoted as a crisp number, can be written as $\widetilde{x}(\alpha)$ in $\{x \mid \tilde{x}(t) \geq \alpha\}$, for $0<\alpha \leq 1$. The interval of the $\alpha$-cut of fuzzy numbers will be written as $\tilde{x}(\alpha)=[\underline{x}(\alpha), \bar{x}(\alpha)]$, for all $\alpha$, since they were always closed and bounded [14]. Suppose $(\underline{x}, \bar{x})$ is parametric form of fuzzy function $x$. For arbitrary positive integer $n$ subdivide the interval $a \leq t \leq b$, whereas $t_{i}=a+i h(i=0,1,2, \ldots, n)$ for $i$ and $h=(b-a) / n$.

Denote the value of $x$ and $(x, \bar{x})$ at the representative point $t_{i}(i=0,1,2, \ldots, n)$ by $x_{i}$ at $\left(x_{i}, \overline{x_{i}}\right)$. Thus, by using the second-order central finite difference scheme, problem (1) can be developed as

$$
\begin{aligned}
& \underline{x_{i}^{\prime \prime}} \approx \frac{x_{i-1}-2 x_{i}+x_{i+1}}{h^{2}}, \\
& \overline{x_{i}^{\prime \prime}} \approx \frac{\overline{x_{i-1}}-2 \overline{x_{i}}+\overline{x_{i+1}}}{h^{2}}, \\
& \overline{x_{i}^{\prime}} \approx \frac{x_{i+1}-x_{i-1}}{2 h} \\
& \overline{x_{i}^{\prime}} \approx \frac{\overline{x_{i+1}}-\overline{x_{i-1}}}{2 h},
\end{aligned}
$$

which give

$$
\begin{aligned}
& x_{i}^{\prime \prime}=\left(\underline{x_{i}^{\prime \prime}}, \overline{x_{i}^{\prime \prime}}\right), \\
& x_{i}^{\prime}=\left(\underline{x_{i}^{\prime}}, \overline{x_{i}^{\prime}}\right) .
\end{aligned}
$$

By using parametric form of fuzzy function, (1) can be written as

$$
\begin{aligned}
& \underline{x_{i}^{\prime \prime}}=\underline{f\left(t_{i}\right)-p\left(t_{i}\right) x_{i}^{\prime}-q\left(t_{i}\right) x_{i}}, \\
& \overline{x_{i}^{\prime \prime}}=\overline{f\left(t_{i}\right)-p\left(t_{i}\right) x_{i}^{\prime}-q\left(t_{i}\right) x_{i}} .
\end{aligned}
$$

Suppose that $p\left(t_{i}\right)>0$ and $q\left(t_{i}\right)>0$ for $i=0,1,2, \ldots, n$. Then

$$
\begin{aligned}
& \underline{x_{i}^{\prime \prime}}+p\left(t_{i}\right) \underline{x_{i}^{\prime}}+q\left(t_{i}\right) \underline{x_{i}}=f\left(t_{i}\right), \\
& \overline{x_{i}^{\prime \prime}}+p\left(t_{i}\right) \overline{x_{i}^{\prime}}+q\left(t_{i}\right) \overline{x_{i}}=f\left(t_{i}\right) .
\end{aligned}
$$

By applying (2a) and (3a), (6a) will be reduced to

$$
\begin{aligned}
& \frac{x_{i-1}-2 x_{i}}{=}+\underline{x_{i+1}}+p\left(t_{i}\right) \frac{x_{i+1}-x_{i-1}}{2 h}+q\left(t_{i}\right) \underline{x_{i}} \\
& =f\left(t_{i}\right)
\end{aligned}
$$

for $i=1,2, \ldots, n-1$. Meanwhile, by substituting (2b) and (3b) into (6b), we will have

$$
\begin{aligned}
& \frac{\overline{x_{i-1}}-2 \overline{x_{i}}+\overline{x_{i+1}}}{h^{2}}+p\left(t_{i}\right) \frac{\overline{x_{i+1}}-\overline{x_{i-1}}}{2 h}+q\left(t_{i}\right) \overline{x_{i}} \\
& =f\left(t_{i}\right) .
\end{aligned}
$$

Then, (7a) and (7b) can be rewritten as follows:

$$
\begin{gathered}
\left(2-h p\left(t_{i}\right)\right) \underline{x_{i-1}}+\left(2 h^{2} q\left(t_{i}\right)-4\right) \underline{x_{i}} \\
+\left(2+h p\left(t_{i}\right)\right) \underline{x_{i+1}}=2 h^{2} f\left(t_{i}\right), \\
\left(2-h p\left(t_{i}\right)\right) \overline{x_{i-1}}+\left(2 h^{2} q\left(t_{i}\right)-4\right) \overline{x_{i}} \\
+\left(2+h p\left(t_{i}\right)\right) \overline{x_{i+1}}=2 h^{2} f\left(t_{i}\right),
\end{gathered}
$$

respectively, for $i=1,2, \ldots, n-1$. Since both of (8a) and (8b) have the same form in terms of the equation, except that, based on the interval of the $\alpha$-cuts, the differences are identified only in the upper and lower bounds, it can be rewritten as

$$
\rho_{i} x_{i-1}+\beta_{i} x_{i}+\varphi_{i} x_{i+1}=F_{i}
$$

for $i=1,2, \ldots, n-1$, where

$$
\begin{aligned}
\rho_{i} & =2-h p\left(t_{i}\right), \\
\beta_{i} & =2 h^{2} q\left(t_{i}\right)-4, \\
\varphi_{i} & =2+h p\left(t_{i}\right), \\
F_{i} & =2 h^{2} f\left(t_{i}\right) .
\end{aligned}
$$

Now, we can express the second-order central finite difference approximation (9) in a matrix form as

$$
A x=b
$$

with

$$
\begin{aligned}
& A=\left[\begin{array}{ccccccc}
\beta_{1} & \varphi_{1} & & & & & \\
\rho_{2} & \beta_{2} & \varphi_{2} & & & & \\
& \rho_{3} & \beta_{3} & \varphi_{3} & & & \\
& & \ddots & \ddots & \ddots & & \\
& & & \rho_{n-3} & \beta_{n-3} & \varphi_{n-3} & \\
& & & & \rho_{n-2} & \beta_{n-2} & \varphi_{n-2} \\
& & & & & \rho_{n-1} & \beta_{n-1}
\end{array}\right]_{(n-1) \times(n-1)}, \\
& x=\left[\begin{array}{lllll}
x_{1} & x_{2} & \cdots & x_{n-2} & x_{n-1}
\end{array}\right]^{T}, \\
& b=\left[\begin{array}{lllll}
f_{1}-\rho_{1} x_{0} & f_{2} & \cdots & f_{n-2} & f_{n-1}-\varphi_{n-1} x_{n}
\end{array}\right]^{T} .
\end{aligned}
$$

Since this study will deal with an application of the method, the computational method of it will be diagonally dominant matrix and positive definite matrix [15].

\section{Two-Parameter Alternating Group Explicit Iterative Method}

Based on previous study conducted by Evans, clearly we can see that they have discussed theoretically how to compute the value of parameter $r$ given by Mohanty et al. [9-13]. In this paper, the optimum value of parameters $r_{1}$ and $r_{2}$ will be 
calculated by implementing several numerical experiments, so those optimum values will be found if the number of iterations is smaller.

Family of AGE can be considered efficient to two-step method to solve linear system. None of the researchers had been trying to apply this method in solving fuzzy problem generated from discretization of fuzzy partial difference equation. This paper will discuss the application of this iterative method which will solve the fuzzy linear system given by (1). Consider a class of methods mentioned in $[4,5]$ which is based on the splitting of the matrix $A$ into the sum of its constituent symmetric and positive definite matrices, as follows:

$$
A=G_{1}+G_{2}
$$

where
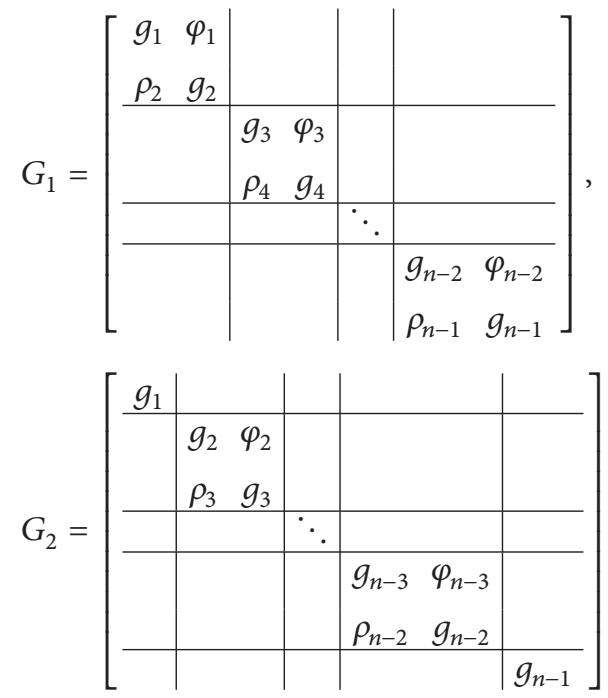

if $n$ is odd. Similarly, we define the following matrices:
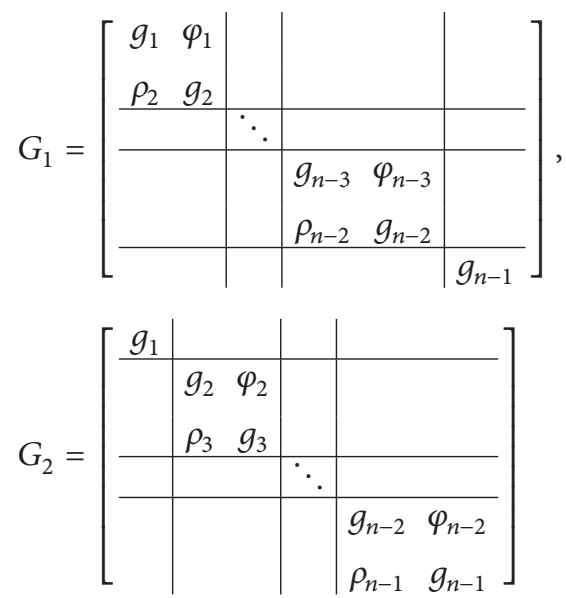

if $n$ is even, with $g_{i}=\beta_{i} / 2(i=1,2, \ldots, n-1)$. In this paper, we only consider that case $n$ is even.

Then (11) becomes

$$
\left(G_{1}+G_{2}\right) x=b
$$

Thus, the explicit form of TAGE method can be written as

$$
\begin{aligned}
x^{(k+1 / 2)} & =\left(G_{1}+r_{1} I\right)^{-1}\left[b-\left(G_{2}-r_{1} I\right) x^{(k)}\right], \\
x^{(k+1)} & =\left(G_{2}+r_{2} I\right)^{-1}\left[b-\left(G_{1}-r_{2} I\right) x^{(k+1 / 2)}\right],
\end{aligned}
$$

where $r_{1}, r_{2}>0$ are the acceleration parameters, and a pair of $\left(G_{1}+r_{1} I\right)$ and $\left(G_{2}+r_{2} I\right)$ are invertible. From (17), therefore, the implementation of TAGE method is presented in Algorithm 1.

Algorithm 1 (TAGE method).

(i) Initialize $\widetilde{U}^{(0)} \leftarrow 0$ and $\varepsilon \leftarrow 10^{-10}$.

(ii) For $i=1 p, 2 p, \ldots, n-p$, initialize parameters $\rho_{i}, \beta_{i}$, $\varphi_{i}, f_{i}, r_{1}, r_{2}, G_{1}$, and $G_{2}$.

(iii) First Sweep. For $i=1 p, 2 p, \ldots, n-p$, compute

$$
x^{(k+1 / 2)}=\left(G_{1}+r_{1} I\right)^{-1}\left[b-\left(G_{2}-r_{1} I\right) x^{(k)}\right] .
$$

(iv) Second Sweep. For $i=1 p, 2 p, \ldots, n-p$, compute

$$
x^{(k+1)}=\left(G_{2}+r_{2} I\right)^{-1}\left[b-\left(G_{1}-r_{2} I\right) x^{(k+1 / 2)}\right] .
$$

(v) Convergence Test. If the convergence criterion, that is, $\left\|\widetilde{U}^{(k+1)}-\widetilde{U}^{k}\right\|_{\infty} \leq \varepsilon$, is satisfied, go to Step (vi). Otherwise go back to Step (ii).

(vi) Display approximate solutions.

\section{Numerical Experiments}

Two examples of FBVPs are considered to verify the effectiveness of GS, AGE, and TAGE methods. For comparison purposes, three parameters were observed that are number of iterations, execution time (in seconds), and Hausdorff distance (as mentioned in Definition 2). Based on these two problems, numerical results for GS, AGE, and TAGE methods have been recorded in Tables 1 to 5 .

Definition 2 (see [16]). Given two minimum bounding rectangles $P$ and $Q$, a lower bound of the Hausdorff distance from the elements confined by $P$ to the elements confined by $Q$ is defined as

$\operatorname{HausDistLB}(P, Q)$

$$
=\operatorname{Max}\left\{\operatorname{MinDist}\left(f_{\alpha}, Q\right): f_{\alpha} \in \text { Faces Of }(P)\right\} .
$$

Problem 1. Consider

$$
x^{\prime \prime}(t)=\widetilde{k}(-6 t), \quad t \in(0,1),
$$

where $\tilde{k}[\alpha]=[\underline{k}(\alpha), \bar{k}(\alpha)]=[0.75+0.25 \alpha, 1.25-0.25 \alpha]$ with the boundary conditions $\tilde{x}(0)=0$ and $\tilde{x}(1)=1$. The exact solutions for

$$
\underline{x^{\prime \prime}}(t ; \alpha)=\underline{k}(\alpha)(-6 t),
$$


TABLE 1: Comparison of three parameters between GS, AGE, and TAGE methods at $\alpha=0.00$.

\begin{tabular}{|c|c|c|c|c|c|c|c|}
\hline & & \multirow{2}{*}{ Methods } & \multicolumn{5}{|c|}{$n$} \\
\hline & & & 512 & 1024 & 2048 & 4096 & 8192 \\
\hline \multirow{9}{*}{ Problem 1} & \multirow{3}{*}{ Number of iterations } & GS & 681711 & 2431928 & 8548735 & 29480437 & 99066551 \\
\hline & & AGE & 96747 & 354438 & 1279808 & 4549671 & 15883620 \\
\hline & & TAGE & 77377 & 279463 & 876061 & 2879619 & 10383345 \\
\hline & \multirow{3}{*}{ Execution time } & GS & 48.94 & 211.19 & 989.91 & 5719.20 & 32465.10 \\
\hline & & AGE & 8.00 & 39.00 & 202.00 & 1310.00 & 8125.00 \\
\hline & & TAGE & 7.00 & 31.00 & 141.00 & 822.00 & 5342.00 \\
\hline & \multirow{3}{*}{ Hausdorff distance } & GS & $2.6560 e-06$ & $1.0624 e-05$ & $4.2497 e-05$ & $1.6999 e-04$ & $6.7995 e-04$ \\
\hline & & AGE & $3.2355 e-07$ & $1.3084 e-06$ & $5.2674 e-06$ & $2.1148 e-05$ & $8.4787 e-05$ \\
\hline & & TAGE & $2.4955 e-07$ & $9.8491 e-07$ & $3.0435 e-06$ & $1.0607 e-05$ & $4.5689 e-05$ \\
\hline \multirow{9}{*}{ Problem 2} & \multirow{3}{*}{ Number of iterations } & GS & 475487 & 1692329 & 5930853 & 20369573 & 68062962 \\
\hline & & AGE & 67638 & 247434 & 891667 & 3161503 & 10997813 \\
\hline & & TAGE & 53492 & 187245 & 671456 & 2122064 & 7505046 \\
\hline & \multirow{3}{*}{ Execution time } & GS & 35.27 & 155.77 & 764.09 & 4457.31 & 26063.40 \\
\hline & & AGE & 6.00 & 27.00 & 141.00 & 912.00 & 5676.00 \\
\hline & & TAGE & 5.00 & 20.00 & 107.00 & 608.00 & 3887.00 \\
\hline & \multirow{3}{*}{ Hausdorff distance } & GS & $2.4952 e-06$ & $7.7115 e-06$ & $3.0279 e-05$ & $1.2097 e-04$ & $4.8386 e-04$ \\
\hline & & AGE & $8.3545 e-07$ & $1.0823 e-06$ & $3.7861 e-06$ & $1.5058 e-05$ & $6.0327 e-05$ \\
\hline & & TAGE & $7.7615 e-07$ & $8.1054 e-07$ & $2.6440 e-06$ & $1.0853 e-05$ & $3.2986 e-05$ \\
\hline
\end{tabular}

TABLE 2: Comparison of three parameters between GS, AGE, and TAGE methods at $\alpha=0.25$.

\begin{tabular}{|c|c|c|c|c|c|c|c|}
\hline & & \multirow{2}{*}{ Methods } & \multicolumn{5}{|c|}{$n$} \\
\hline & & & 512 & 1024 & 2048 & 4096 & 8192 \\
\hline \multirow{9}{*}{ Problem 1} & \multirow{3}{*}{ Number of iterations } & GS & 682475 & 2434982 & 8560953 & 29529307 & 99262033 \\
\hline & & AGE & 96840 & 354815 & 1281323 & 4555751 & 15908020 \\
\hline & & TAGE & 77449 & 279746 & 876948 & 2882382 & 10399116 \\
\hline & \multirow{3}{*}{ Execution time } & GS & 49.07 & 211.36 & 991.23 & 5874.81 & 32551.12 \\
\hline & & AGE & 9.00 & 39.00 & 202.00 & 1301.00 & 8164.00 \\
\hline & & TAGE & 7.00 & 31.00 & 141.00 & 827.00 & 5402.00 \\
\hline & \multirow{3}{*}{ Hausdorff distance } & GS & $2.6560 e-06$ & $1.0624 e-05$ & $4.2497 e-05$ & $1.6999 e-04$ & $6.7995 e-04$ \\
\hline & & AGE & $3.2355 e-07$ & $1.3083 e-06$ & $5.2675 e-06$ & $2.1148 e-05$ & $8.4786 e-05$ \\
\hline & & TAGE & $2.4955 e-07$ & $9.8490 e-07$ & $3.0417 e-06$ & $1.0452 e-05$ & $4.5526 e-05$ \\
\hline \multirow{9}{*}{ Problem 2} & \multirow{3}{*}{ Number of iterations } & GS & 476030 & 1694502 & 5939547 & 20404350 & 68202066 \\
\hline & & AGE & 67704 & 247701 & 892745 & 3165828 & 11015151 \\
\hline & & TAGE & 53543 & 187435 & 672208 & 2124610 & 7514448 \\
\hline & \multirow{3}{*}{ Execution time } & GS & 35.26 & 155.79 & 756.06 & 4465.35 & 25999.98 \\
\hline & & AGE & 6.00 & 27.00 & 142.00 & 903.00 & 5652.00 \\
\hline & & TAGE & 5.00 & 21.00 & 106.00 & 605.00 & 3893.00 \\
\hline & \multirow{3}{*}{ Hausdorff distance } & GS & $2.4650 e-06$ & $7.7039 e-06$ & $3.0277 e-05$ & $1.2097 e-04$ & $4.8386 e-04$ \\
\hline & & AGE & $8.0517 e-07$ & $1.0748 e-06$ & $3.7841 e-06$ & $1.5058 e-05$ & $6.0327 e-05$ \\
\hline & & TAGE & $7.4595 e-07$ & $8.0323 e-07$ & $2.6424 e-06$ & $1.0749 e-05$ & $3.2626 e-05$ \\
\hline
\end{tabular}

$$
\overline{x^{\prime \prime}}(t ; \alpha)=\bar{k}(\alpha)(-6 t)
$$

are

$$
\begin{aligned}
& \underline{x}(t ; \alpha)=\underline{k}(\alpha)\left[-t^{3}+2 t\right], \\
& \bar{x}(t ; \alpha)=\bar{k}(\alpha)\left[-t^{3}+2 t\right],
\end{aligned}
$$

respectively.
Problem 2 (see [17]). Consider

$$
x^{\prime \prime}(t)-4 x(t)=\widetilde{k}(4 \cosh (1)), \quad t \in(0,1),
$$

where $\tilde{k}[\alpha]=[\underline{k}(\alpha), \bar{k}(\alpha)]=[0.75+0.25 \alpha, 1.25-0.25 \alpha]$ with the boundary conditions $\tilde{x}(0)=0$ and $\tilde{x}(1)=0$. The exact solutions for

$$
\underline{x^{\prime \prime}}(t ; \alpha)-4 \underline{x}(t ; \alpha)=\underline{k}(\alpha)(4 \cosh (1)),
$$


TABLE 3: Comparison of three parameters between GS, AGE, and TAGE methods at $\alpha=0.50$.

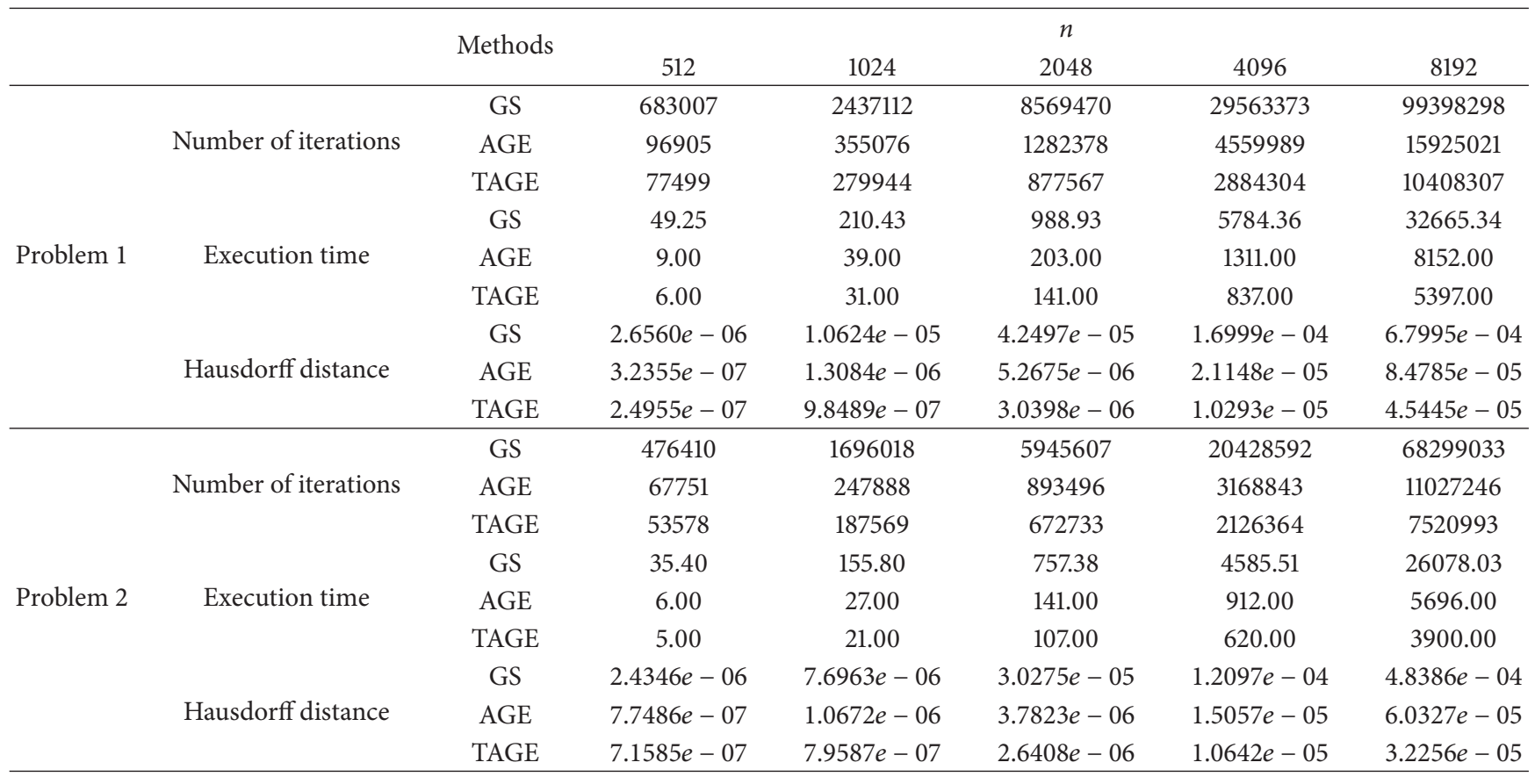

TABLE 4: Comparison of three parameters between GS, AGE, and TAGE methods at $\alpha=0.75$.

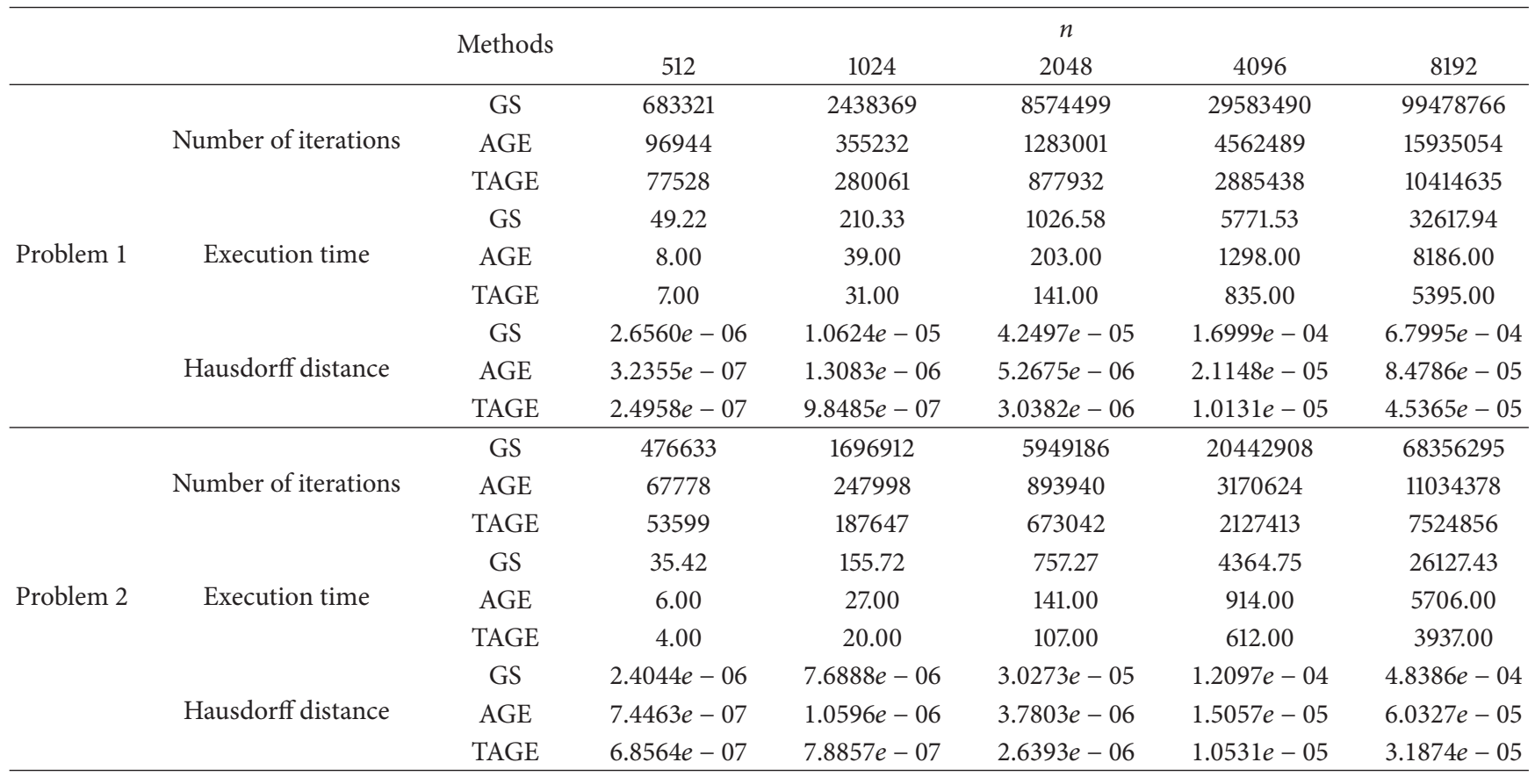

$$
\overline{x^{\prime \prime}}(t ; \alpha)-4 \bar{x}(t ; \alpha)=\bar{k}(\alpha)(4 \cosh (1))
$$

are

$$
\begin{aligned}
& \underline{x}(t ; \alpha)=\underline{k}(\alpha)[\cosh (2 t-1)-\cosh (1)], \\
& \bar{x}(t ; \alpha)=\bar{k}(\alpha)[\cosh (2 t-1)-\cosh (1)],
\end{aligned}
$$

respectively.

\section{Conclusions}

In this paper, TAGE method was used to solve linear systems which arise from the discretization of two-point FBVPs using the second-order central finite difference scheme. The results show that TAGE method is more superior in terms of the number of iterations, execution time, and Hausdorff distance compared to the AGE and GS methods. Since TAGE is well suited for parallel computation, it can be considered as a main 
TABLE 5: Comparison of three parameters between GS, AGE, and TAGE methods at $\alpha=1.00$.

\begin{tabular}{|c|c|c|c|c|c|c|c|}
\hline & & \multirow{2}{*}{ Methods } & \multicolumn{5}{|c|}{$n$} \\
\hline & & & 512 & 1024 & 2048 & 4096 & 8192 \\
\hline \multirow{9}{*}{ Problem 1} & \multirow{3}{*}{ Number of iterations } & GS & 683426 & 2438784 & 8576162 & 29590144 & 99505380 \\
\hline & & AGE & 96956 & 355282 & 1283208 & 4563320 & 15938400 \\
\hline & & TAGE & 77538 & 280098 & 878054 & 2885812 & 10416768 \\
\hline & \multirow{3}{*}{ Execution time } & GS & 49.45 & 210.66 & 809.53 & 5758.67 & 32519.13 \\
\hline & & AGE & 9.00 & 39.00 & 202.00 & 1313.00 & 8221.00 \\
\hline & & TAGE & 7.00 & 31.00 & 141.00 & 817.00 & 5383.00 \\
\hline & \multirow{3}{*}{ Hausdorff distance } & GS & $2.6559 e-06$ & $1.0624 e-05$ & $4.2497 e-05$ & $1.6999 e-04$ & $6.7995 e-04$ \\
\hline & & AGE & $3.2354 e-07$ & $1.3084 e-06$ & $5.2674 e-06$ & $2.1148 e-05$ & $8.4783 e-05$ \\
\hline & & TAGE & $2.4955 e-07$ & $9.8492 e-07$ & $3.0366 e-06$ & $9.9651 e-06$ & $4.5321 e-05$ \\
\hline \multirow{9}{*}{ Problem 2} & \multirow{3}{*}{ Number of iterations } & GS & 476706 & 1697208 & 5950370 & 20447642 & 68375230 \\
\hline & & AGE & 67786 & 248034 & 894086 & 3171216 & 11036748 \\
\hline & & TAGE & 53606 & 187674 & 673146 & 2127768 & 7526132 \\
\hline & \multirow{3}{*}{ Execution time } & GS & 35.43 & 155.72 & 755.20 & 4615.31 & 25815.45 \\
\hline & & AGE & 6.00 & 27.00 & 141.00 & 915.00 & 5662.00 \\
\hline & & TAGE & 4.00 & 21.00 & 107.00 & 613.00 & 3941.00 \\
\hline & \multirow{3}{*}{ Hausdorff distance } & GS & $2.3742 e-06$ & $7.6812 e-06$ & $3.0271 e-05$ & $1.2097 e-04$ & $4.8386 e-04$ \\
\hline & & AGE & $7.1441 e-07$ & $1.0521 e-06$ & $3.7785 e-06$ & $1.5056 e-05$ & $6.0326 e-05$ \\
\hline & & TAGE & $6.5552 e-07$ & $7.8117 e-07$ & $2.6376 e-06$ & $1.0417 e-05$ & $3.1481 e-05$ \\
\hline
\end{tabular}

advantage because this method has groups of independent task which can be implemented simultaneously. It is hoped that the capability of the proposed method will be helpful for the further investigation in solving any multidimensional fuzzy partial differential equations [18]. Basically the results of this paper can be classified as one of full-sweep iteration. Apart from the concept of the full-sweep iteration, further investigation of half-sweep [19-24] and quarter-sweep [2527] iterations can also be considered in order to speed up the convergence rate of the standard proposed iterative methods. Other than that, further study will be extended to solve nonlinear problem by combining Newton-Raphson method.

\section{Conflict of Interests}

The authors declare that there is no conflict of interests regarding the publication of this paper.

\section{Acknowledgment}

This paper was funded by National Defence University of Malaysia.

\section{References}

[1] J. J. Buckley and T. Feuring, "Fuzzy differential equations," Fuzzy Sets and Systems, vol. 110, no. 1, pp. 43-54, 2000.

[2] N. Gasilov, S. E. Amrahov, and A. G. Fatullayev, "Linear differential equations with fuzzy boundary values," in Proceedings of the 5th International Conference on Applied Information and Communication Technology, 2011.

[3] S. Seikkala, "On the fuzzy initial value problem," Fuzzy Sets and Systems, vol. 24, no. 3, pp. 319-330, 1987.
[4] K. S. Sukon, "On two parameter alternating group explicit (TAGE) method for singular perturbation problems," Parallel Algorithms and Applications, vol. 10, no. 1-2, pp. 71-77, 1996.

[5] K. S. Sukon and D. J. Evans, “Two parameter age (TAGE) method for the solution of a tradiagonal linear system of equations," International Journal of Computer Mathematics, vol. 60, no. 3-4, pp. 265-278, 1996.

[6] D. J. Evans, "The alternating group explicit (AGE) matrix iterative method," Applied Mathematical Modelling, vol. 11, no. 4, pp. 256-263, 1987.

[7] D. J. Evans, Group Explicit Methods for the Numerical Solution of Partial Differential Equations, Gordon and Breach Science Publishers, 1997.

[8] N. Jha and R. K. Mohanty, "TAGE iterative algorithm and nonpolynomial spline basis for the solution of nonlinear singular second order ordinary differential equations," Applied Mathematics and Computation, vol. 218, no. 7, pp. 3289-3296, 2011.

[9] R. K. Mohanty, M. K. Jain, and D. Dhall, "A cubic spline approximation and application of TAGE iterative method for the solution of two point boundary value problems with forcing function in integral form," Applied Mathematical Modelling, vol. 35, no. 6, pp. 3036-3047, 2011.

[10] R. K. Mohanty and D. Dhall, "Third order accurate variable mesh discretization and application of TAGE iterative method for the non-linear two-point boundary value problems with homogeneous functions in integral form," Applied Mathematics and Computation, vol. 215, no. 6, pp. 2024-2034, 2009.

[11] R. K. Mohanty and N. Khosla, "Application of TAGE iterative algorithms to an efficient third order arithmetic average variable mesh discretization for two-point non-linear boundary value problems," Applied Mathematics and Computation, vol. 172, no. 1, pp. 148-162, 2006.

[12] R. K. Mohanty, P. L. Sachdev, and N. Jha, "An An $\mathrm{O}\left(h^{4}\right)$ accurate cubic spline TAGE method for nonlinear singular 
two point boundary value problems," Applied Mathematics and Computation, vol. 158, no. 3, pp. 853-868, 2004.

[13] R. K. Mohanty, P. L. Sachdev, and N. Jha, "TAGE method for nonlinear singular two point boundary value problem using a fourth order difference scheme," Neural, Parallel \& Scientific Computations, vol. 11, no. 3, pp. 281-296, 2003.

[14] T. Allahviranloo, "Difference methods for fuzzy partial differential equations," Computational Methods in Applied Mathematics, vol. 2, no. 3, pp. 233-242, 2002.

[15] D. M. Young, "Second-degree iterative methods for the solution of large linear systems," Journal of Approximation Theory, vol. 5, pp. 137-148, 1972.

[16] S. Nutanog, H. E. Jacox, and H. Samet, "An incremental Hausdorff distance calculation algorithm," Proceedings of the VLDB Endowment, vol. 4, pp. 506-517, 2011.

[17] A. Mohsen and M. El-Gamel, "On the Galerkin and collocation methods for two-point boundary value problems using sinc bases," Computers \& Mathematics with Applications, vol. 56, no. 4, pp. 930-941, 2008.

[18] A. Farajzadeh, A. Hossein Pour, and M. Amini, "An explicit method for solving fuzzy partial differential equation," International Mathematical Forum, vol. 5, no. 21-24, pp. 1025-1036, 2010.

[19] A. A. Dahalan, J. Sulaiman, and M. S. Muthuvalu, "Performance of HSAGE method with Seikkala derivative for 2-D fuzzy Poisson equation," Applied Mathematical Sciences, vol. 8, no. 1720, pp. 885-899, 2014.

[20] A. A. Dahalan, M. S. Muthuvalu, and J. Sulaiman, "Numerical solutions of two-point fuzzy boundary value problem using half-sweep alternating group explicit method," in International Conference on Mathematical Sciences and Statistics 2013, vol. 1557 of AIP Conference Proccedings, pp. 103-107, 2013.

[21] M. Sundaram Muthuvalu and J. Sulaiman, "Half-sweep geometric mean iterative method for the repeated Simpson solution of second kind linear Fredholm integral equations," Proyecciones. Journal of Mathematics, vol. 31, no. 1, pp. 65-79, 2012.

[22] M. S. Muthuvalu and J. Sulaiman, "Half-sweep arithmetic mean method with composite trapezoidal scheme for solving linear Fredholm integral equations," Applied Mathematics and Computation, vol. 217, no. 12, pp. 5442-5448, 2011.

[23] J. Sulaiman, M. Othman, and M. K. Hasan, "Half-sweep algebraic multigrid (HSAMG) method applied to diffusion equations," in Modeling, Simulation and Optimization of Complex Processes, pp. 547-556, Springer, Berlin, Germany, 2008.

[24] J. Sulaiman, M. K. Hasan, and M. Othman, “The half-sweep iterative alternating decomposition explicit (HSIADE) method for diffusion equation," in Computational and Information Science, J. Zhang, J.-H. He, and Y. Fu, Eds., vol. 3314 of Lecture Notes in Computer Science, pp. 57-63, Springer, Berlin, Germany, 2005.

[25] N. Jha, "The application of sixth order accurate parallel quarter sweep alternating group explicit algorithm for nonlinear boundary value problems with singularity", in Proceedings of the International Conference on Methods and Models in Computer Science (ICM2CS '10), pp. 76-80, IEEE, 2010.

[26] J. Sulaiman, M. Othman, and M. K. Hasan, "A new quartersweep arithmetic mean (QSAM) method to solve diffusion equations," Chamchuri Journal of Mathematics, vol. 1, no. 2, pp. 89-99, 2009.

[27] J. Sulaiman, M. Othman, and M. K. Hasan, "Quarter-sweep iterative alternating decomposition explicit algorithm applied to diffusion equations," International Journal of Computer Mathematics, vol. 81, no. 12, pp. 1559-1565, 2004. 


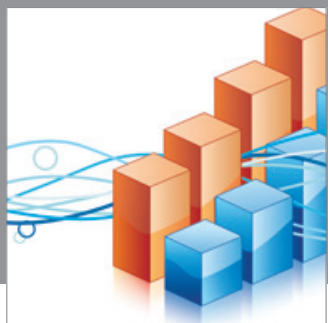

Advances in

Operations Research

mansans

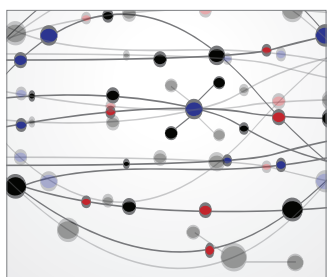

The Scientific World Journal
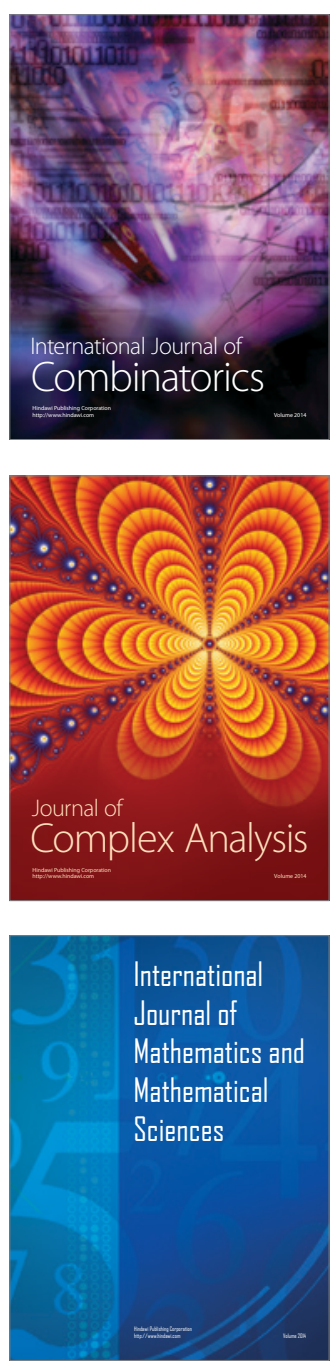
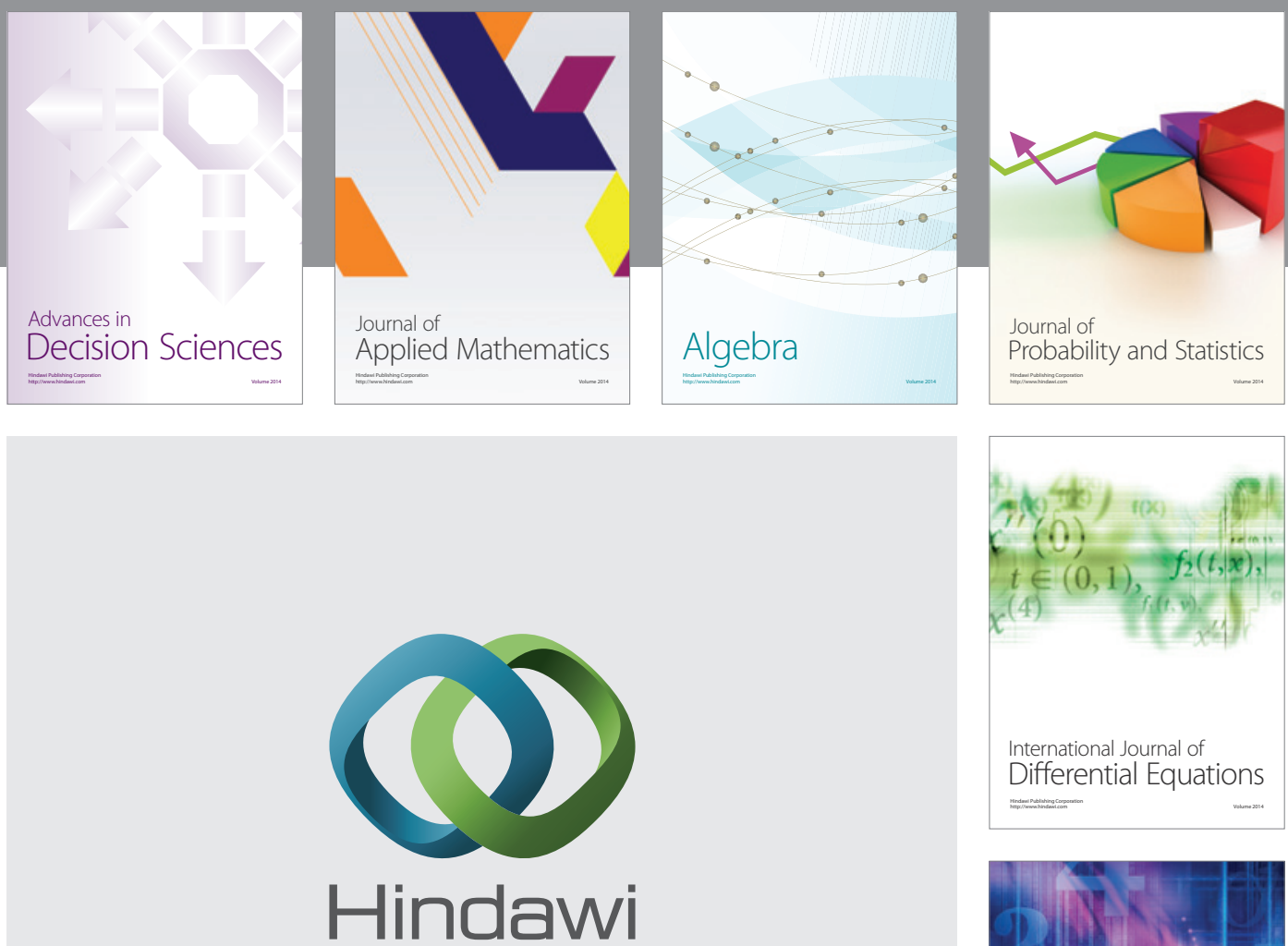

Submit your manuscripts at http://www.hindawi.com
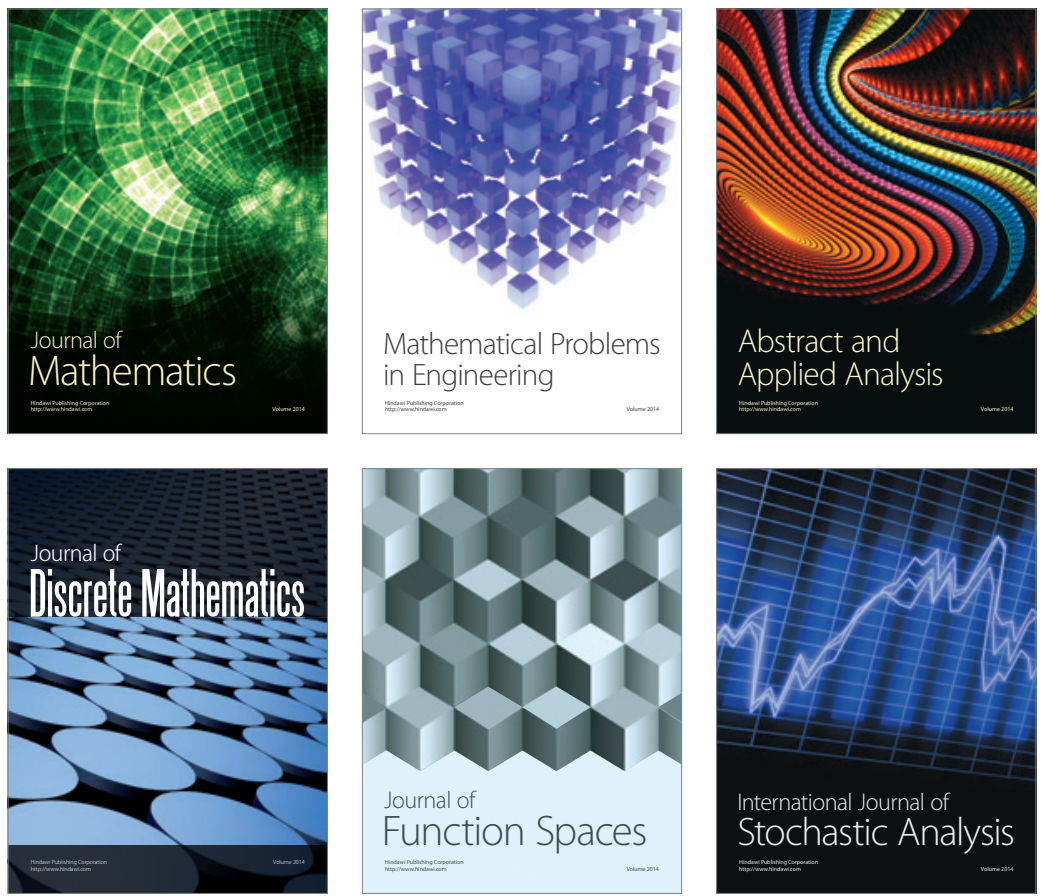

Journal of

Function Spaces

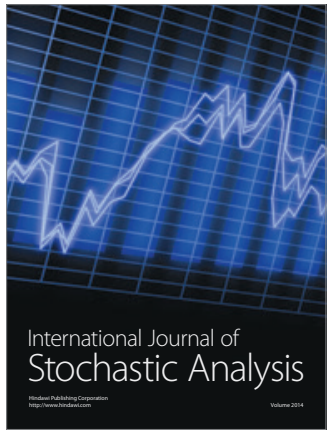

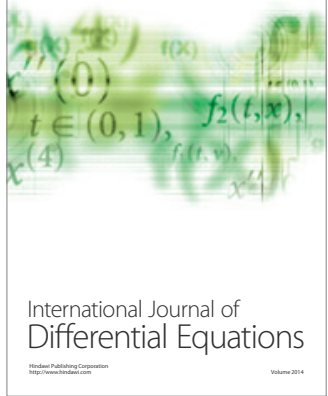
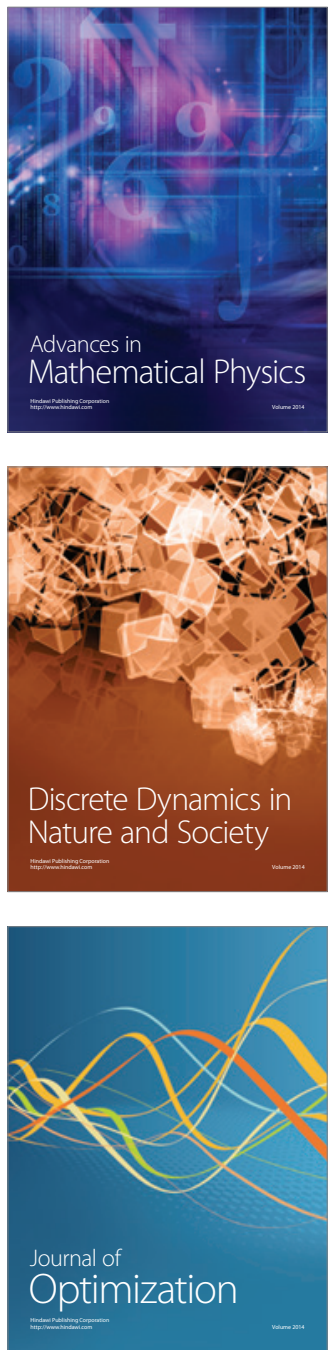